\title{
Finding Organized Structures in 3-D Ladar Data
}

\author{
Nicolas Vandapel and Martial Hebert \\ The Robotics Institute \\ Carnegie Mellon University \\ Pittsburgh, PA, USA
}

\begin{abstract}
In this paper, we address the problem of finding organized thin structures in three-dimensional (3-D) data. Linear and planar structures segmentation received much attention but thin structures organized in complex patterns remain a challenge for segmentation algorithms. We are interested especially in the problems posed by repetitive and symmetric structures acquired with a laser range finder. The method relies on 3-D data projections along specific directions and 2-D histograms comparison. The sensitivity of the classification algorithm to the parameter settings is evaluated and a segmentation method proposed. We illustrate our approach with data from a concertina wire in terrain with vegetation.
\end{abstract}

\section{INTRODUCTION}

Classification and segmentation of natural scenes from three-dimensional (3-D) laser data received much attention from the research community for tasks such as scene interpretation, obstacle detection or terrain-based localization [1], [2], [3], [4]. A substantial body of work exists on how to extract planar and linear objects from scattered 3-D point clouds, see for example [5], [6]. Methods were even proposed to detect solid surfaces hidden inside foliage [7]. But small scale structures, such as wires and thin poles, are still challenging for segmentation algorithms.

In this paper, we look at the detection and segmentation of organized thin structures. In our terminology, thin structures differ from linear structures in the following ways: linear structures are elongated, locally smooth set of points (typically large branches or posts) while thin structures are elongated and a few pixels wide (typically small branches or wires). Organized thin structures are complex patterns of thin structures. There are two key issues in detecting such structures: 1) the small number of points on the target of interest, and 2) their structural similarity with vegetation and foliage. We are interested in characterizing such structure by building a discriminative local structure signature. We focus our effort on a special class of organized thin structures: periodic and symmetric structures. Through out the paper we will illustrate our approach in the context of detecting a concertina wire in vegetated terrains using data collected with an actuated SICK laser range finder. Figure 1 shows a representative scene containing vegetation and a concertina wire.

Our proposed local structure signature relies on data reduction, both in dimension and in size, by projecting and accumulating 3-D data into different 2-D sub-spaces. The approach is inspired by the work on local shape representation with Spin-Images by Johnson [8] and with Shape Contexts by Belongie [9]. The level of organization

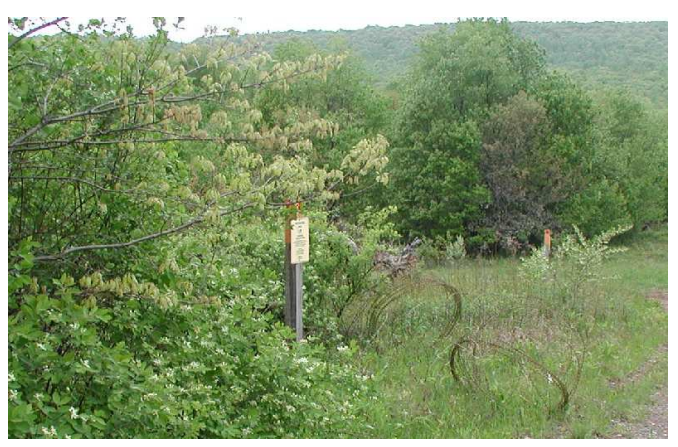

Fig. 1. Representative terrain of interest containing vegetation and a concertina wire

of the point cloud in which we are interested is quantified by an absolute measure of local bilateral symmetry.

The rest of the paper is composed of four sections. In the next section we review current methods on sensing and segmentation of thin structures. In Section III, we present the details of our approach. In Section IV, we illustrate the performances of the method on real data before concluding.

\section{RELATED WORK}

\section{A. Sensing}

The two closest related fields in thin structure detection is power line detection for aircraft obstacle avoidance at low altitude and stand-off trip wire detection for landmine clearance. Laser-based obstacle detection systems are commercially available for power line detection on-board manned helicopters. Typically, they are designed to detect at $600 \mathrm{~m}$ cables with diameter of several centimeters, stretching in a linear fashion between towers with no clutter. The emphasis is on early and reliable detection and not on 3-D reconstruction [10]. Other sensing modalities tested include millimeter wave radar [11] and passive infrared camera [12]. Trip-wires, used in some landmines, can be detected, using passive polarimetric infrared sensors [13] or active illumination with short-wave infrared laser [14]. Those wires are a few millimeters in diameter and cluttered by vegetation.

The accurate 3-D reconstruction of thin structures is very difficult in natural cluttered settings such as the ones encountered by ground mobile robots. Thin structures will produce mixed pixels that are difficult to compensate for using methods based on local smoothness of the scene [15]. 


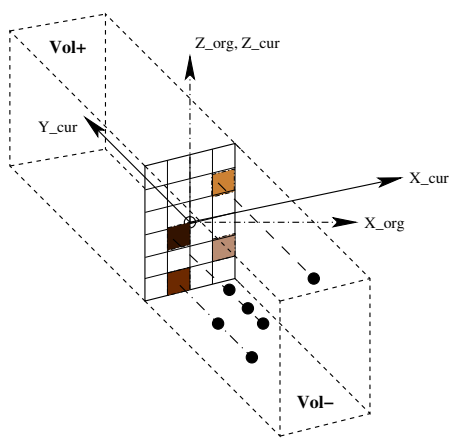

(a)

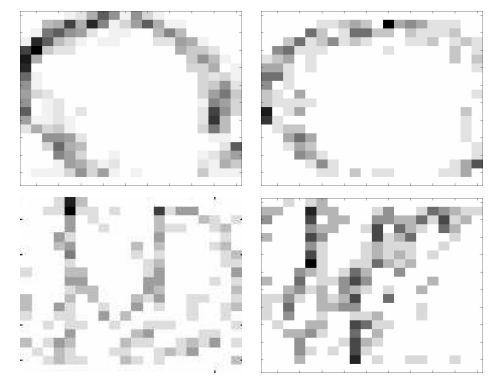

(b)

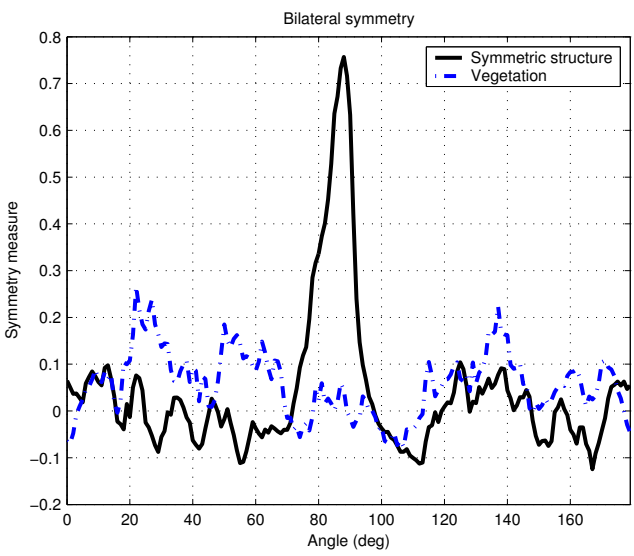

(c)

Fig. 2. (a) Projection for bilateral symmetry detection. (b) Example of data from a concertina wire projected into two plane configurations: the top/bottom 2-D histograms correspond to the plane orientation with the highest/lowest score, the right/left 2-D histograms correspond to $H_{+}$and $H_{-}$. (c) Symmetry measure for a location with a symmetric structure and a random structure.

\section{B. Segmentation}

In this paper, we address the problem of structure detection and segmentation from 3-D point clouds collected from a single sensor location or integrated from multiple locations. In [2], Lacaze proposed to use the permeability of the scene to laser beam to discriminate between solid surfaces (ground, obstacle) and vegetation. This method is popular for outdoor robot navigation [16], [1], [17] but does not allow object segmentation or scene structures inference. Methods that fit primitives to point clouds are difficult to use practically for large data sets containing multiple complex structures, in opposition to multiple planar surfaces that can be handled. Similar methods using a single range image are sensitive to occlusion. In addition they focus on piece-wise smooth structures, not on porous objects such as a concertina wire. Tensor voting was proven to be a powerful tool to segment surface, curve and junction from 3-D data [6]. We follow a similar approach in [18], as described in the next section. Tubular structures from veins can be extracted from dense 3-D volumetric medical data [19]. Data that differs very much from the sparse discontinuous data we work with.

\section{Our previous approach}

In [18], we proposed a method to classify 3-D ladar data in natural terrain into three classes: vegetation, solid surface and linear structure. The method estimates the local point distribution in space and uses a Bayes classifier to produce the probability of belonging to each class. Priors are modeled as Mixtures of Gaussians and parameters are learned using the Expectation-Maximization algorithm (EM) on labelled data. At each point, the scatter matrix is computed using a predefined support region. The principal components of this matrix are used to define three saliency features for each scale [6], characterizing the 3-D points' spatial distribution into the three classes.

This method gives good results with thin linear objects with low curvature. Multiple thin linear objects close to each other, intersecting or forming junctions are more challenging. Thin structures forming a compact pattern such as a coil fall into that category. Figure 6-(c) shows an example where a complex symmetric structure cannot be detected reliably with such a method. The scene contains a concertina wire cluttered by vegetation. Most of the points from the structure are classified as scatter (green points) while few are as linear structure (blue points) as we would like to see. In the next section we present a method to address this problem.

\section{APPROACH}

\section{A. Structure characterization}

Our approach is inspired by the work of Kazhdan et al. in [20]. The authors propose to use a reflective symmetry descriptor to build a signature of a 3-D object that can be used for evaluating object similarity. Our approach differs in a number of areas. The authors deal with scenes made of a unique complete object (no missing data, no clutter). We are dealing with partial data of an object of interest submerged in clutter. The authors deal with object comparison, we are dealing with structure detection and then segmentation. In addition we do not compare against a data base model. Several methods have been proposed to detect reflective/bilateral symmetry [21], [22], [23], [24]. We decided to follow the approach of [8], [9] which projects the data into a different subspace to perform comparison.

\section{B. Dimension reduction}

The core of the method is to project and accumulate the 3-D points of the scene into a gridded planar surface to produce a 2-D histogram for each orientation of the plane. Figure 2-(a) illustrates the geometry of the projection. For each orientation, two 2-D histograms $\left(H_{+}\right.$and $\left.H_{-}\right)$are produced corresponding to the projection of the points in the two volumes ( $\mathrm{Vol}_{+}$and $\mathrm{Vol}_{-}$) separated by the projection plane. The method is characterized by the following parameters: the width $(\mathrm{W})$ and height $(\mathrm{H})$ of the planar area where points are projected, the maximum distance to 


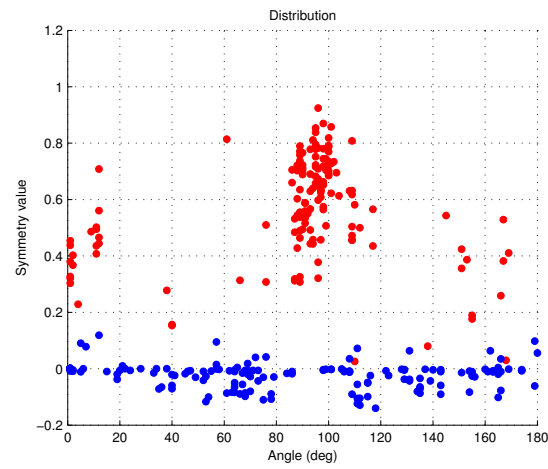

(a) A concertina wire

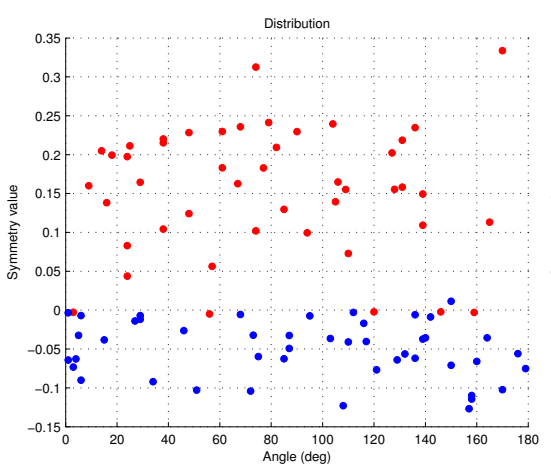

(b) Foliage

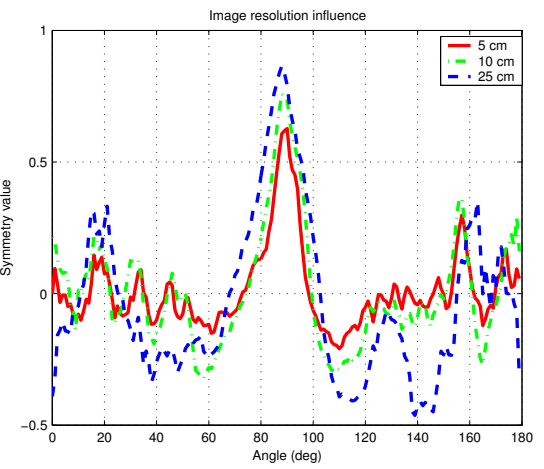

(c) Projection plane lattice resolution influence

Fig. 3. (a)-(b) Dominant symmetry direction and amplitude computed for multiple locations from a concertina wire and foliage data. In red the most likely symmetric direction and in blue the less likely. (c) Projection plane lattice resolution influence. Symmetry measure for one location, with a constant support region but with different projection cell resolution.

the plane for a point to be projected $\left(d_{\text {proj }}^{\max }\right)$, the number of bins in the projection plane $\left(n_{w} \times n_{h}\right)$ and the size of the bins $\left(s_{w} \times s_{h}\right)$, the location of the center of the projection plane $\left(O_{p}\right)$, and finally the axis $(A)$ and the angle $(\theta)$ of rotation of the plane. In this paper the axis $A$ is aligned with the vertical. Figure 2-(b) shows two examples of 2-D histograms computed for the same location (center of the coil) but for different plane orientations. The projection plane contains $20 \times 20$ square bins, $5 \mathrm{~cm}$ wide. $d_{\text {proj }}^{\max }$ is set to 1 meter. The darker the pixel is, the higher the density of points in the bin is.

A measure of bilateral symmetry is naturally computed by comparing the two 2-D histograms $H_{+}$and $H_{-}$. Several distance measures, such as histogram intersection or the Earth Mover's Distance [25], can be considered. Here we use the normalized correlation, denoted $R\left(H_{+}, H_{-}\right)$with $R \in[-1,1]$, which was used by Johnson in [8] with similar signatures.

By computing this symmetry value for different plane orientations we obtain a curve $R=f(\theta)$. Figure 2-(c) shows two examples: one from a concertina wire data and the other from random data. The absolute maximum of the curve determines the orientation and the intensity of the dominant local bilateral symmetry of scene.

In the next section we review the properties of the method, we present experimental results on wire detectability, and results on a concertina wire detection in cluttered vegetated terrain.

\section{IMPLEMENTATION AND EXPERIMENTATIONS}

\section{A. Properties}

We now review five properties of the symmetry measure presented above: scale, angular resolution, discrimination against noise, projection plane resolution influence, and finally robustness against clutter.

1) Scale: Symmetry is a scale dependent property of objects. The scale is defined by the size of the projection area $(\mathrm{W} \times \mathrm{H})$ and the range of projection $\left(d_{p r o j}^{\max }\right)$. They are defined to capture the structure of the object of interest. In the example presented in this paper we choose $d_{\text {proj }}^{\max }$ equal to 1 or $1.25 \mathrm{~m}$ and a one meter square projection area.
2) Angular resolution: In the examples presented so far the symmetry measure is computed for every angle of the rotation plane, producing 180 values per location. In practice, the curves are smooth and it is therefore possible to evaluate the symmetry for a sparse set of orientation, thus speeding up the computation. In the example presented in Figure 6-(d) the angle interval is $4^{\circ}$.

3) Discrimination against random points: In Figure 3, we present results showing that the signature can discriminate between an organized points cloud from a concertina wire and scattered points from foliage. For multiple locations, symmetry curves are computed and the absolute extrema are extracted. Figures 3-(a) and 3-(b) show their distribution for a concertina wire and foliage respectively. In Figure 3-(a) points are clustered around the direction $90^{\circ}$ corresponding to the plane normal to the main axis of the coil. In Figure 3-(b) the data is distributed randomly. Note the difference of symmetry intensity between the two graphs.

4) 2-D histogram resolution: Figure 3-(c) shows the influence of the projection plane resolution on the symmetry value. We use data from a concertina wire. The range of influence and the size of the projection area are maintained constant, set both at $1 \mathrm{~m}$. Three pixel resolutions are used 5,10 and $25 \mathrm{~cm}$. As the resolution decreases, new modes appear in the graph $\left(118^{\circ}\right.$ and $\left.142^{\circ}\right)$, the symmetry value increases but the value of the dominant symmetry mode remains at the same location $\left(90^{\circ}\right)$ and its maximum well above the mean value.

5) Clutter: One critical property for the approach to be applicable in vegetated terrain is the ability of the signature to handle clutter around the structure of interest. We compared the symmetry curve, for two projection plane lattice resolutions, for a concertina wire without, and with different levels of clutter simulated using a Poisson distribution, following [26]. Figure 4-(a) shows a top view of the scene corrupted with noise. The other graphs in Figure 4 show the symmetry curve for the same location, for the same range $(1 \mathrm{~m})$, the same projection plane size $(1 \mathrm{~m} \times 1 \mathrm{~m})$ but at two different resolutions $(10 \times 10$ and $20 \times 20$ ). Note that, in each graph, the curves are similar. 


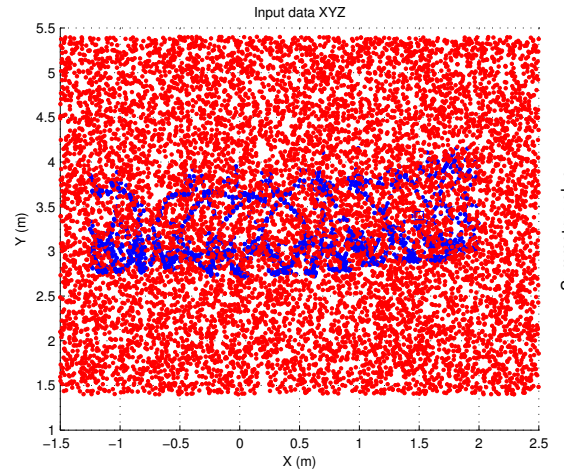

(a) Scene top view

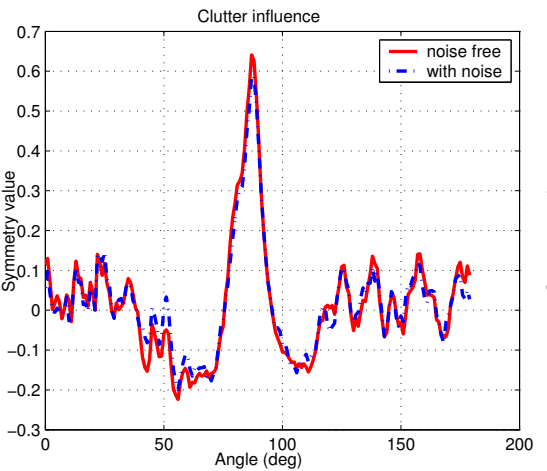

(b) $20 \times 20$ pixels 2-D histogram

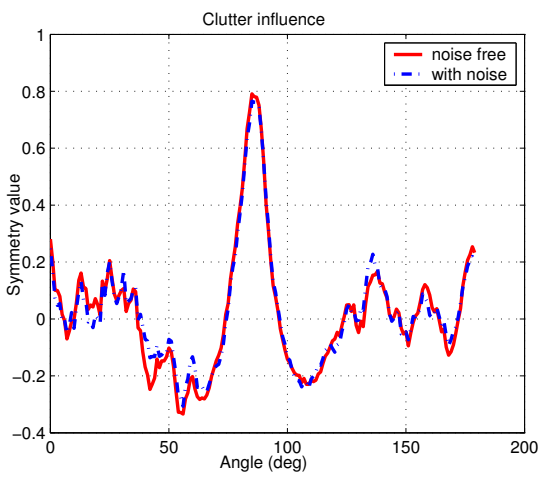

(c) $10 \times 10$ pixels 2-D histogram

Fig. 4. Clutter influence on the symmetry measure. (a) Scene with in red/blue the scene/added noise data (b)-(c) Symmetry values compute for the same location at low and high resolution. Each graph is made of the superposition of several plot corresponding to increasing level of noise.

The scene contains 2388 points from the concertina. The volume occupied is $2.5 \mathrm{~m} \times 1 \mathrm{~m} \times 1 \mathrm{~m}$. The three levels of noise considered correspond to 100, 150 and 200 noise points per cubic meter.

\section{B. Configuration space exploration}

In our current implementation, the intensity and direction of the bilateral symmetry is computed on a 3-D lattice spaced every half to one meter. Figure 5 shows an example of such a result. In this top view of the scene of an isolated coil of wires, ladar points are in red, the lattice points are in green and the direction and intensity of the symmetry is represented as a blue vector. Note the change of orientation of the main axis of the coil correctly captured by the direction of the blue lines. The break point is located at $(0.25,3.25)$. The ground data points are filtered out before processing.

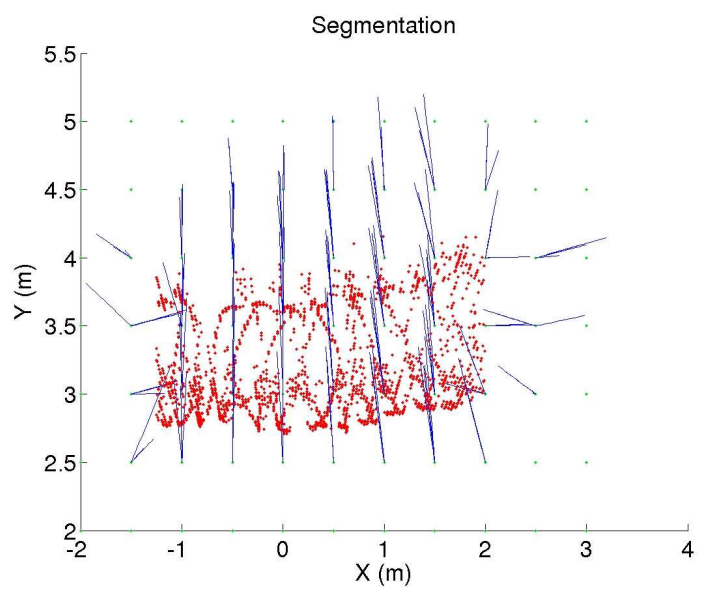

Fig. 5. Top view of the scene. The red points are the ladar data, the green points are the location where the symmetry has been evaluated, in blue, vectors representing the orientation and the intensity of the bilateral symmetry.

\section{Performance analysis}

1) Sensing issues: We used an actuated SICK LMS291-SO5 mounted on a cart to collect 3-D scans. We performed a test to determine the detectability of a concertina wire ${ }^{1}$ using the highest angular resolution setting of the scanner. With such setting the laser produce $400 \times 800$ points for a field of view of $100^{\circ} \times 120^{\circ}$. Results are presented in Table I. The coil structure is preserved up to 8 meters in the configuration tested.

\section{TABLE I}

WIRE DETECTABILITY BY THE ACTUATED SICK LASER AT LOW ANGULAR RESOLUTION. THE RANGE IS IN METER.

\begin{tabular}{l|lrrrrrrrrrr} 
Range & 2.6 & 3.6 & 4.6 & 5.6 & 6.8 & 7.6 & 8.6 & 9.6 & 10.6 & 11.6 & 12.6 \\
\hline Points & 2506 & 1695 & 1178 & 820 & 521 & 389 & 219 & 171 & 138 & 98 & 72
\end{tabular}

2) Scene segmentation: We collected data sets of the coil positioned in a vegetated terrain, seen from different viewpoints and with different level of clutter. Figure 6-(a) is an image of the scene. Results from the processing of two data sets are presented here: normal incidence with clutter (Figure $6-(\mathrm{a} / \mathrm{b} / \mathrm{c} / \mathrm{d})$ ) and oblique view angle with no clutter (Figure 6-(e)/(f)). The laser is positioned at 7 $\mathrm{m}$ from the coil and the scanner is set in high resolution mode. For space consideration we decided not to present the corresponding Figure 6-(b/c) for the oblique view.

The data was processed in three steps: 1) ground separation, 2) non-ground data classification in three classes (clutter, linear, surface), 3) dominant bilateral symmetry detection. The ground data is separated by using the method proposed in [4] and the result is seen in Figure 6-(b). The classification results of the step 2 can be seen in 6-(c). Notice that it is classified partially as linear and partially as vegetation. We mentioned this problem earlier in Section II-C. Figure 6-(d) shows the distribution of the dominant symmetry direction and value for an area surrounding the coil. The symmetry measure is calculated every four degrees. The coil contributes to the accumulation around the $22^{\text {nd }}$ bin.

We produced similar results with the oblique view of the scene (Figure-(e/f)). Figure 6-(e) is a top view of the scene. The red points are the ladar data, the green points are the

\footnotetext{
${ }^{1}$ The concertina wire used is made of a $3.5 \mathrm{~mm}$ diameter cable forming a $1 \mathrm{~m}$ diameter coil. Small blades $(10 \mathrm{~mm} \times 13 \mathrm{~mm})$ are locate every $2.6 \mathrm{~cm}$ along the cable.
} 
locations where the symmetry has been evaluated, in blue, vectors representing the orientation and the intensity of the bilateral symmetry. The distribution of the same results is presented 6-(f). The concertina wire can be detected by clustering the data in the symmetry value, direction feature space. In this example, the coil is adjacent to but not cluttered by vegetation unlike in the previous example.

\section{CONCLUSiON}

In this paper, we propose a method to detect organized thin structures in 3-D ladar data. This work is a new contribution of our effort in [4] to reliably segment vegetation terrain for ground mobile robot navigation. We consider a symmetric structure made of wires configured to form a concertina pattern. The method allows the recovery of the local dominant bilateral symmetry direction and intensity. We show the influence of the method parameters using data from a controlled environment. We also show segmentation results of the concertina wire in vegetated terrain.

Future work will address the characterization of additional gross structures in addition to the current bilateral symmetry. Statistically significant evaluations on various scenes are planned.

\section{ACKNOWLEDGMENTS}

Prepared through collaborative participation in the Robotics Consortium sponsored by the U.S Army Research Laboratory under the Collaborative Technology Alliance Program, Cooperative Agreement DAAD19-01209912. We would like to thank Ranjith Unnikrishnan for helping collecting the data.

\section{REFERENCES}

[1] A. Stentz, A. Kelly, P. Rander, H. Herman, O. Amidi, R. Mandelbaum, G. Salgian, and J. Pedersen, "Real-time, multi-perspective perception for unmanned ground vehicles," in AUVSI, 2003.

[2] A. Lacaze, K. Murphy, and M. Delgiorno, "Autonomous mobility for the demo iii experimental unmanned veh icles," in Proceedings of the AUVSI Conference, 2002.

[3] A. Talukder, R. Manduchi, A. Rankin, and L. Matthies, "Fast and reliable obstacle detection and segmentation for cross-count ry navigation," in IEEE Intelligent Vehicle Symposium, 2002.

[4] N. Vandapel, R. R. Donamukkala, and M. Hebert, "Experimental results in using aerial ladar data for mobile robot navigation," in International Conference on Field and Service Robotics, 2003.

[5] R. Unnikrishnan and M. Hebert, "Robust extraction of multiple structures from non-uniformly sampled data," in IEEE/RSJ International Conference on Intelligent Robots and System, 2003.

[6] C.-K. T. Gerard G. Medioni, Mi-Suen Lee, A Computational Framework for Segmentation and Grouping. Elsevier, 2000.
[7] A. Castano and L. Matthies, "Foliage discimination using a rotating ladar," in IEEE International Conference on Robotics and Automation, 2003.

[8] A. Johnson, "Spin-images: A representation for 3-d surface matching," Ph.D. dissertation, Robotics Institute, Carnegie Mellon University, Pittsburgh, PA, August 1997.

[9] S. Belongie, J. Malik, and J. Puzicha, "Shape matching and object recognition using shape contexts," in IEEE Transactions on Pattern Analysis and Machine Intelligence, 2001.

[10] K. R. Schultz, S. Scherbarth, and U. Fabry, "Hellas: Obstacle warning system for helicopters," in Laser Radar Technology and Applications VII, vol. 4723. SPIE, 2002.

[11] Y.-H. Kim and G.-S. Kang, "Multi-wire detection and image reconstruction using $27 \mathrm{ghz}$ isar," in IEEE International Geoscience and Remote Sensing Symposium, 1999.

[12] K. Yamamoto and K. Yamada, "Analysis of the infrared images to detect power lines," in TENCON, 1997.

[13] G. Forssell, "Passive ir polarization models and measurements applied to covered surface landmines and trip wires," in Detection and Remediation Technologies for Mines and Minelike Targets VIII, 2003.

[14] T. Allik, D. Dawkins, K. Sherbondy, and J. Nettleton, "Eyesafe laser illuminated tripwire (elit) detector," in Detection and Remediation Technologies for Mines and Minelike Targets VI, vol. 4394. SPIE, 2001.

[15] M. Adams, "On-line gradient based surface discontinuity detection for outdoor scanning range sensors," in IEEE/RSJ International Conference on Intelligent Robots and System, 2001.

[16] M. Ollis and T. M. Jochem, "Structural method for obstacle detection and terrain classification," in Unmanned Ground Vehicle Technology, 2003.

[17] C. Wellington and A. Stentz, "Learning predictions of the loadbearing surface for autonomous rough-terrain navigation in vegetation," in International Conference on Field and Service Robotics, 2003.

[18] N. Vandapel, D. Huber, A. Kapuria, and M. Hebert, "Natural terrain classification using 3-d ladar data," in IEEE International Conference on Robotics and Automation, 2004.

[19] A. chung and J. noble, "Statistical 3d vessel segmentation using a rician distribution," in Medical Image Understanding and Analysis Conference, 1999.

[20] M. Kazhdan, B. Chazelle, D. Dobkin, A. Kinkelstein, and T. F nkhouser, "A reflective symmetry descriptor," in European Conference on Computer Vision, 2002.

[21] G. D. Stetten and S. M. Pizer, "Medial-node models to identify and measure objects in real-time $3 \mathrm{~d}$ ea chocardiography," IEEE Transactions on Medical Imaging, vol. 18, no. 10, 1999.

[22] Y. Liu, R. T. Collins, and Y. Tsin, "A computational model for periodic pattern perception based on frieze and wallpaper groups," IEEE Transactions on Pattern Analysis and Machine Intelligence, vol. 26, no. 3, 2003.

[23] D. Shen, H. Ip, K. Cheung, and E. Teoh, "Symmetry detection by generalized complex (gc) moments: a close-form solution," IEEE Transactions on Pattern Analysis and Machine Intelligence, 1999.

[24] C. Sun and J. Sherrah, "3d symmetry detection using the extended gaussian image," IEEE Transactions on Pattern Analysis and Machine Intelligence, 1997.

[25] Y. Rubner, C. Tomasi, and L. J. Guibas, "The earth mover's distance," International Journal of Computer Vision, 2000.

[26] J. Huang, A. Lee, and D. Mumford, "Statistics of range images," in IEEE International Conference on Pattern Recognition, 2000. 


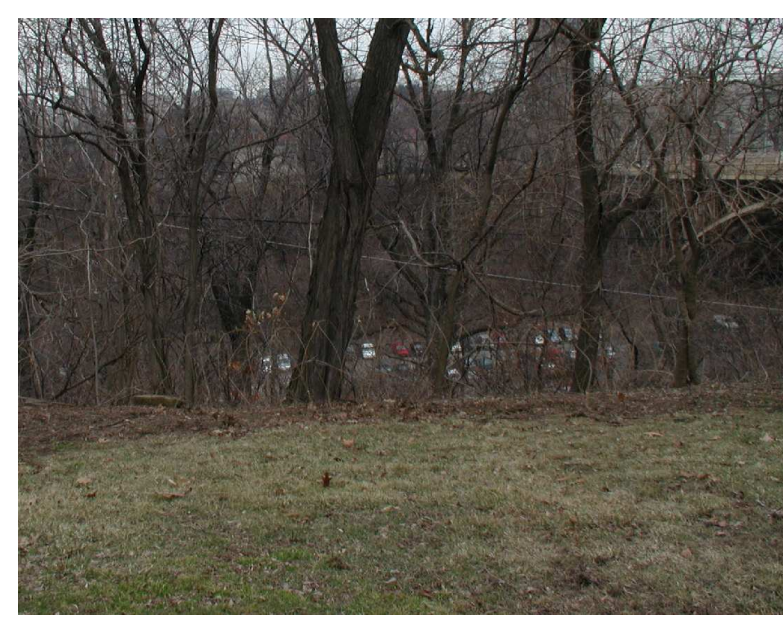

(a) Scene

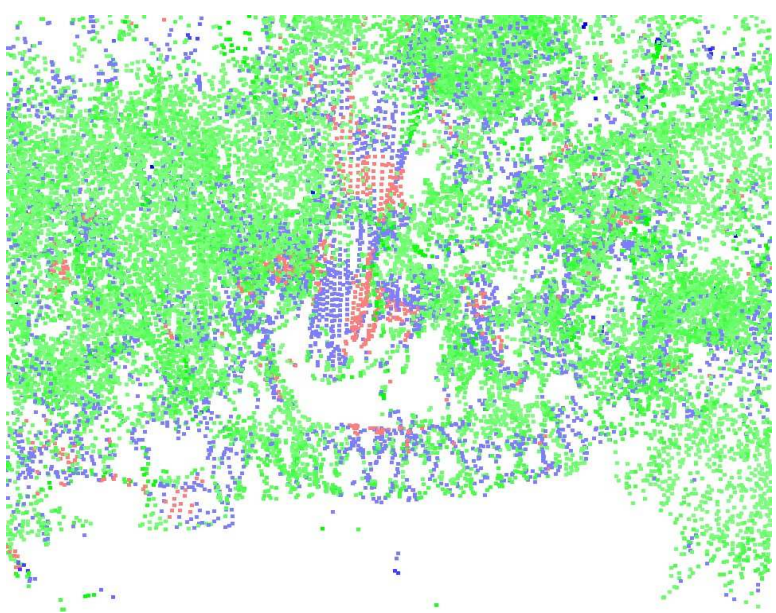

(c) Segmentation of non-ground points into linear/scatter/surface classes following [18]

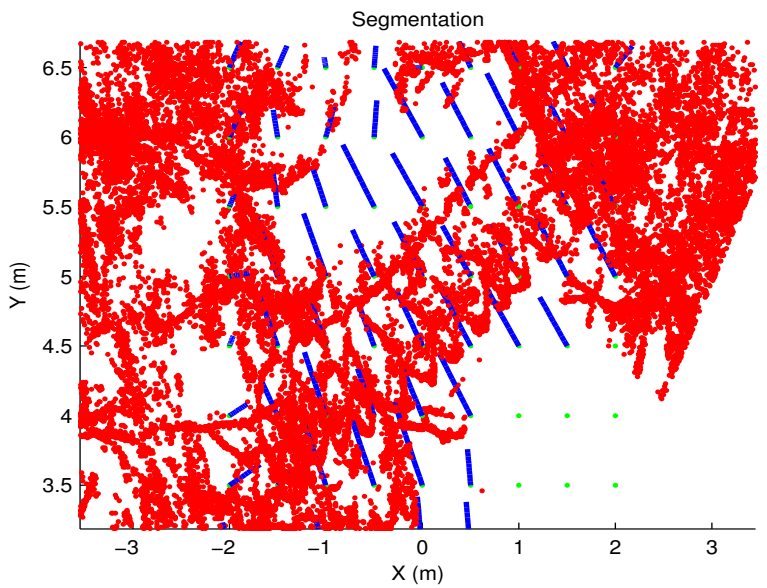

(e) Symmetry detection

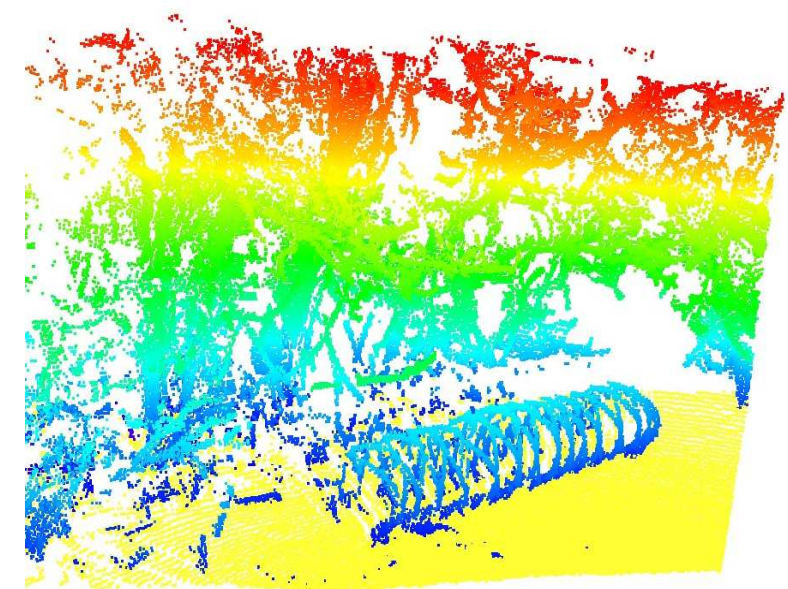

(b) Scene segmented into ground/non ground

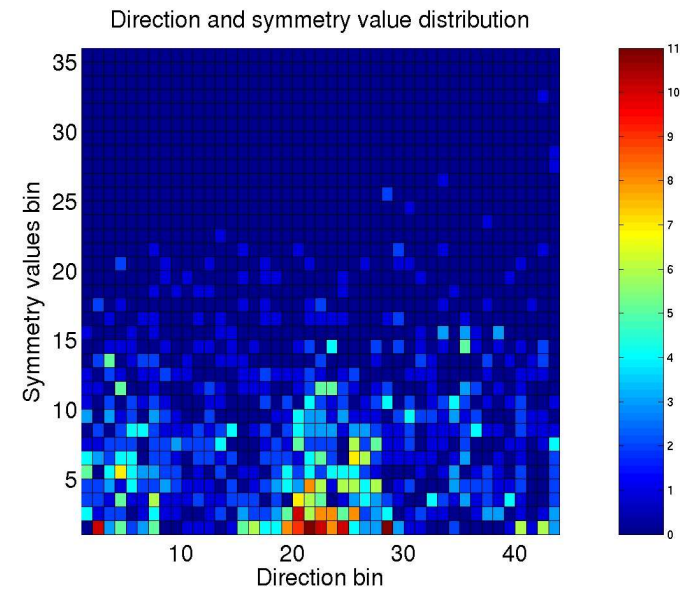

(d) Symmetry detection

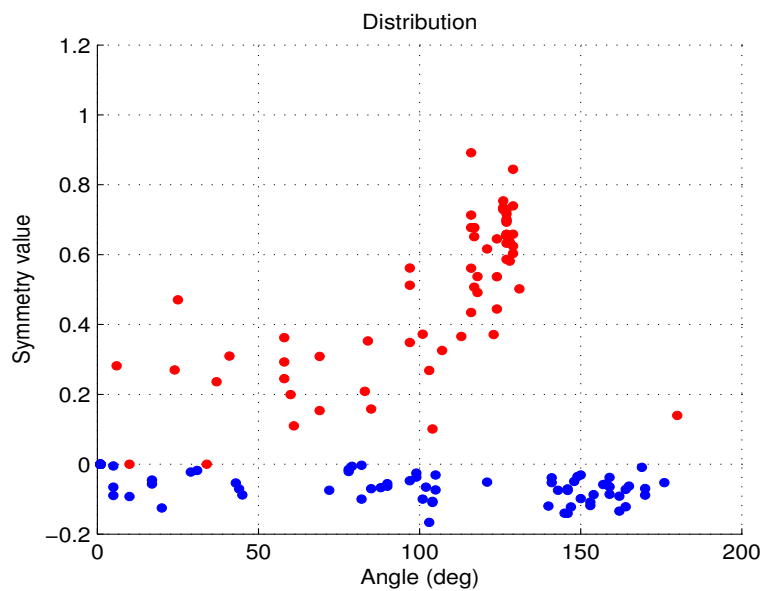

(f) Symmetry value and orientation distribution

Fig. 6. Example of scene analysis. (a) The scene: concertina wire against vegetation with branches added to clutter the wires. (b) Segmentation of the ground points represented in light brown color. The rest of the points are colored based on their elevation. (c) Segmentation of the non-ground points into linear (blue), scatter (green) and solid (red) structures. (d) Symmetry direction and intensity distribution computed at locations selected randomly in the scene but below one meter elevation from the ground. Normal incidence data collection. (e) Top view of the scene. The red points are the ladar data, the green points are the location where the symmetry has been evaluated, in blue, vectors representing the orientation and the intensity of the bilateral symmetry (f) Dominant symmetry direction and amplitude computed for multiple locations from the concertina wire and foliage data. In red the most likely symmetric direction and in blue the less likely. (e)/(f) produced using the side view data collection. 